\title{
Erratum to: Practical assessment of preoperative functional mapping techniques: navigated transcranial magnetic stimulation and functional magnetic resonance imaging
}

\author{
Antonella Mangraviti - Cecilia Casali - Roberto Cordella - Federico Giuseppe Legnani • \\ Luca Mattei · Francesco Prada • Andrea Saladino • Valeria Elisa Contarino • \\ Alessandro Perin · Francesco DiMeco
}

Published online: 1 May 2013

(C) Springer-Verlag Italia 2013

\section{Erratum to: Neurol Sci}

DOI 10.1007/s10072-012-1283-7

Owing to a technical error, the author Valeria Elisa Contarino was inadvertently missed from the list of authors. The correct author group is given below.

Author group:

Antonella Mangraviti - Cecilia Casali · Roberto Cordella · Federico Giuseppe Legnani · Luca Mattei · Francesco Prada · Andrea Saladino - Valeria Elisa Contarino - Alessandro Perin · Francesco DiMeco

Valeria Elisa Contarino

Neuroradiology, Fondazione IRCCS Istituto Neurologico C. Besta, Milan, Italy

The online version of the original article can be found under doi:10.1007/s10072-012-1283-7.

\footnotetext{
A. Mangraviti - C. Casali - R. Cordella - F. G. Legnani - L. Mattei ·

F. Prada · A. Saladino - A. Perin · F. DiMeco $(\square)$

Neurosurgery, Fondazione IRCCS Istituto Neurologico C. Besta,

Milan, Italy

e-mail: fdimeco@istituto-besta.it

L. Mattei

Department of Neurosurgery, Università degli Studi di Milano,

Milan, Italy

V. E. Contarino

Neuroradiology, Fondazione IRCCS Istituto Neurologico

C. Besta, Milan, Italy

F. DiMeco

Department of Neurosurgery, Johns Hopkins University,

Baltimore, MD, USA
}

The article also featured an incomplete record of the acknowledgments. The correct version should read as:

"We are grateful to the Tekmed Instruments S.p.A Milan for leasing the Nexstim NBS System 4.0 free of charge; special thanks go to engineers Silvia Marelli and Marco Romiti for their professional assistance". The co-authors apologize for the inconvenience to all concerned. 